\title{
Comparing Programming Self-Esteem of Upper Secondary School Teachers to CS1 Students
}

\author{
Roisin Faherty \\ Department of Computing \\ TU Dublin \\ Dublin, Ireland \\ Roisin.Faherty@TUDublin.ie \\ Monica M. McGill \\ Knox College \& CSEdResearch.org \\ Galesburg, Illinois, USA \\ monica@csedresearch.org
}

\author{
Keith Quille \\ Department of Computing \\ TU Dublin \\ Dublin, Ireland \\ Keith.Quille@TUDublin.ie \\ Brett A. Becker \\ School of Computer Science \\ University College Dublin \\ Dublin, Ireland \\ Brett.Becker@ucd.ie
}

\author{
Rebecca Vivian \\ School of Computer Science \\ The University of Adelaide \\ Adelaide, South Australia, Australia \\ Rebecca.Vivian@adelaide.edu.au \\ Karen Nolan \\ Dept. of Computing \\ TU Dublin \\ Dublin, Ireland \\ Karen.Nolan@TUDublin.ie
}

\begin{abstract}
Teacher self-esteem has been found to impact student learning in a number of non-computing fields. As computing slowly becomes a part of the upper secondary school (high school) curriculum in many countries, instruments designed to measure teachers' programming self-esteem can help inform classroom practice and processes such as teacher professional development needs. This study examines if there are differences in programming self-esteem (using the Bergin Programming Self-Esteem Instrument) between upper secondary school teachers and CS1 students in Ireland. In addition this study provides evidence of validity when using this instrument (originally developed for CS1 students) to measure upper secondary school teacher programming self-esteem.

To test for evidence of validity, we compared the results of the programming self-esteem construct given to upper secondary school teachers $(n=130)$ to a recent study of programming selfesteem among CS1 students $(n=693)$. We found evidence of both reliability and validity with teachers that aligns with the evidence found for the CS1 students, demonstrating utility for use with teacher cohorts.

Comparing these findings, teachers reported statistically significantly lower programming self-esteem compared to CS1 students. Interestingly CS1 students identifying as male had a statistically significant higher programming self-esteem than those identifying as female. However, we found no statistically significant difference for teacher gender, unlike previous work. Our results indicate that teacher programming self-esteem should be given consideration in the design and implementation of professional development.
\end{abstract}

\section{KEYWORDS}

CS1; introductory programming; professional development; K-12 teachers; self-esteem

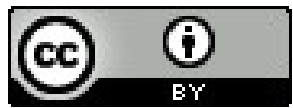

This work is licensed under a Creative Commons Attribution International 4.0 License. ITiCSE 2021, June 26-fuly 1, 2021, Virtual Event, Germany.

(C) 2021 Copyright held by the owner/author(s).

ACM ISBN 978-1-4503-8214-4/21/06.

https://doi.org/10.1145/3430665.3456372
ACM Reference Format:

Roisin Faherty, Keith Quille, Rebecca Vivian, Monica M. McGill, Brett A. Becker, and Karen Nolan. 2021. Comparing Programming Self-Esteem of Upper Secondary School Teachers to CS1 Students. In 26th ACM Conference on Innovation and Technology in Computer Science Education V. 1 (ITiCSE 2021), June 26-July 1, 2021, Virtual Event, Germany. ACM, New York, NY, USA, 7 pages. https://doi.org/10.1145/3430665.3456372

\section{INTRODUCTION}

Teacher self-esteem is known to impact student academic achievement and motivation in non-computing fields [28, 33, 48]. Selfesteem is closely related to, and influences, self-efficacy [3, 48], which is an individual's own beliefs regarding how well one can execute courses of action required to deal with prospective situations [2, p. 122]. Unlike self-efficacy, which is more task-specific, self-esteem is an affective evaluation of one's self $[16,29,51]$. That is, self-efficacy might relate to a teacher's belief on how they teach computing in their curriculum, self-esteem measure might relate to a statement such as 'I feel that I do not have much computer science ability to be proud of' [11]. This is related to sense of belonging, which measures how accepted one feels in a community. Sense of belonging has been shown to vary with many factors in computing students [34].

Several instruments have been created to measure explicit selfesteem in students; however, instruments to measure self-esteem in the context of computing are not numerous [20, 30, 47]. In 2006, Bergin developed a scale to measure programming self-esteem among introductory programming students [8]. In this paper we use the term CS1 as a synonym for introductory programming as is common in third-level computing education [7].

This study investigates the validity of the Bergin Programming Self-Esteem Instrument to measure teacher programming self-esteem. Criterion related validity evaluates the extent to which an instrument or scale in the instrument predicts a variable that is designated as a criterion-or its outcome [17, 32]. There are two forms of criterion validity-concurrent and predictive. Predictive validity measures how likely the scale can be used to predict a future related variable (like success in programming) and concurrent validity measures the scale against other similar scales or across population groups in the same field. In the latter, the comparison is made 
against results that have been determined to measure that construct in either the same or a different context, location, or culture.

In addition to exploring the validity of this instrument with teachers, we investigate the following research question: How do results of the Bergin Programming Self-Esteem Instrument differ between upper secondary school computing teachers and a previous administration of the instrument with CS1 students? Comparing these may help inform future professional development (PD) curriculum. For instance, if an institution had based teacher PD on their staple CS1 course, or encouraged teachers to take a tertiary CS1 course, but it is determined that teachers have higher (or lower) programming self-esteem than tertiary students, the approach may be ill suited. This study is important for designers of a PD curriculum as well as others interested in teacher programming self-esteem and how it may compare to undergraduate students still learning CS1. It is also important for those interested in using the same programming self-esteem scale in computing education research studies as well as for evaluating teacher pre- and post-PD.

\section{BACKGROUND}

In this section, we provide an overview of related work involving self-esteem in computing for both undergraduate students and teachers. We also describe the instrument used to measure programming self-esteem of CS1 students and the implementation of a programming self-esteem instrument in teacher professional development (PD). Since very few instruments or scales exist to measure self-esteem in computing we also include work on the related construct of self-efficacy.

\subsection{Measuring Self-Esteem in Computing}

2.1.1 Students. Self-esteem and self-efficacy in education, as it pertains to students, has been examined over the last several decades. In CS, several instruments have been used to measure self-efficacy in the context of computing. However, searching for an instrument to measure self-esteem tailored to computing, we were able to find only one by Hippler [24].

In 2018, Hippler examined the self-esteem of computing undergraduate women majoring in computing using an instrument based on the Rosenberg Self-Esteem Questionnaire [24, 47]. Hippler found that for these women, general feelings of self-esteem were higher than their self-esteem within computing and there was a correlation between self-esteem and academic standing. Interestingly, Hippler also found that although student answers to competence questions were higher, their worthiness questions were lower, indicating that despite understanding concepts, they valued this knowledge differently. Training can change such views however, and Hippler explores this further in other work [25].

Although no other self-esteem instruments could be found within the context of computing for students, we examined a few instruments measuring self-efficacy, a closely related factor. In 2003, Quade developed a 21-item scale for measuring perceptions of capability associated with success in CS0 students, the Computer Science Self-Efficacy Scale for CSO Courses [35]. CS0 is an introductory programming (not just basic computer literacy) course either for non-majors or those in need of extra preparation before CS1 [23].
Closely modelled after Bandura's scale of self-efficacy, Quade provides evidence of validity for this scale via results of a principal factor analysis. The author further suggests incorporating specific activities and assignments within the course to build confidence, which may help these students succeed further.

In 2011, Blouin created and administered the Computer Science Interest Survey, which was designed to measure computer self-efficacy as well as interest in computing careers [13]. Reliability of the selfefficacy scale was found to be $\alpha=0.77$. Though no significant differences were found between the self-efficacy of genders of high school students, a positive relationship was found between selfefficacy and students who had taken at least one computing course while in high school.

Drobnis (2010) created a new instrument, Attitudes about Computers and Computer Science, designed to measure understanding of students' thoughts, preconceptions, attitude, knowledge of computer science, and future intentions around computer science, both in education and career [22]. The 6-item self-efficacy scale had evidence of face validity, but no other details, (such as internal consistency) were provided. Results of four specific items indicated not only that self-efficacy was lower among females, but also that after a course in computing, females' scores of self-efficacy increased to a greater extent than the increase among males.

In 1994, Torkzadeh and Koufteros used factor analysis and Cronbach's $\alpha$ to demonstrate evidence of reliability and validity of the Personal Computer Self-Efficacy Belief Scale $(\alpha=0.96)$ [52]. When conducting a study using this instrument, they found that training participants in the study on computers significantly improved the computer self-efficacy of both males and females across all factors.

In 1996, Busch measured reliability of the Computer Self-Efficacy Scale using Cronbach's $\alpha$ [14]. With respect to self-efficacy, $\alpha$ was found to be 0.74 . The primary study using this instrument found that female undergraduate students (majoring in business) had a significantly lower self-efficacy in computing, had less previous computer experience, and had received less previous encouragement to work with computers than males.

In 1998, Ramalingam and Wiedenbeck created the Computer Programming Self-Efficacy Scale to measure self-efficacy for computer programming [46]. The scale, primed for $\mathrm{C}++$, was shown to have evidence of reliability and validity using exploratory factor analysis for validity with oblimin rotation which yielded four factors. Cronbach's $\alpha$ was 0.94 for the independence/persistence factor, 0.93 for complex programming tasks, 0.86 for self-regulation, and 0.93 for simple programming tasks, for an overall reliability score of $\alpha=0.98$. Test-rest alpha reliability was 0.97 . In a pre-test post-test study, no significant differences were found between male and female students who participated in the pilot development of the scale.

2.1.2 Teachers. We found few results on self-esteem for teachers. In a 1996 qualitative study on pre-service teachers, Dobbins found that self-esteem was a factor involved in learning [21]. As expected, self-esteem was a variable that changed regularly based on the participants' personal stress factors, energy levels, and the support received during student teaching. That being said, self-esteem still had an impact on their teaching, coping skills, abilities to interact with their students, and how they learned about teaching in general. 
Despite finding only minimal studies on self-esteem of teachers, we found several recent instruments that measure self-efficacy among upper secondary school teachers who teach computing. In 2015, Bean et al., created the Teachers' Self-Efficacy in Computational Thinking (TSECT) instrument which was designed to measure pre-service teachers' self-efficacy in both programming and computational thinking within their future teaching roles with evidence of reliability and validity [4]. Further, the initial study using the instrument demonstrated a strong correlation between improved self-efficacy with computational thinking and with their mastery experiences using computational thinking embedded within other subject areas.

In 2018, Vivian and Falkner [53] created a survey instrument based on Bandura's self-efficacy scale [3] to measure teachers' selfefficacy with respect to teaching and assessing the Australian Curriculum: Digital Technologies, aligned with the Australian Teacher Professional Standards. In the context of the curriculum initiatives in Australia, the study findings suggested that although secondary school teachers reported higher self-efficacy levels in most categories than primary teachers, overall, teachers reported moderate levels of self-efficacy. Additionally, the findings suggest that the farther along teachers were in implementing the various phases of curriculum, the higher the self-efficacy levels. Another study in 2018 by Mannila, et al. [31], measured the self-efficacy of teachers with respect to digital technologies. Findings suggest that self-efficacy in teachers is highest in the area of information and data literacy and lowest for programming.

\subsection{The BPSEI Instrument}

The Bergin Programming Self-Esteem Instrument (BPSEI) was developed as part of a longitudinal study conducted in 2002-2006 [8, 11]. The survey instrument was one of several factors used to predict students early in their CS1 course who were at risk of failing or dropping out. Programming self-esteem was found to be the strongest factor when predicting students at risk of failing or dropping out of CS1, and positively correlated with success.

Bergin modified the Rosenberg [47] self-esteem questionnaire to apply specifically to programming self-esteem. The Rosenberg selfesteem scale is perhaps the most widely used self-esteem measure in social science research. The 10 -item scale has been shown to have generally high inter-item and test-retest reliability evidence [47]. Each question was modified to relate to programming. For example, the first question was changed from "On the whole, I am satisfied with myself" to "On the whole, I am satisfied with my programming progress" [37]. The complete scale is presented in Table 1.

Principal Component Analysis (PCA) was used to reduce the Bergin Programming Self-Esteem Instrument (BPSEI) to one value to be used in the prediction model [43]. PCA essentially performs an orthogonal transformation (rotation of data in multi-dimensions) to find the covariance eigenvectors with the largest eigenvalues which represented the largest distribution or effect of the data set, hence selecting the principal component [6]. The original work by Bergin used the first principal component which almost always accounts for the vast majority of variance, thus producing a single value for programming self-esteem, which is useful for comparisons and analysis.
Table 1: The Bergin programming Self-Esteem Scale consists of 10 items on a 5 -point Likert scale $(1 \rightarrow$ Strongly Disagree, $5 \rightarrow$ Strongly Agree).

\section{Items}

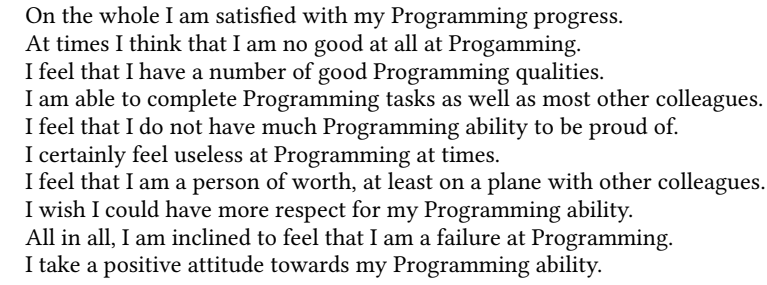

The BPSEI instrument was a core component in a study that spanned over a decade (2006-2017) [9, 15, 19, 36-43]. Once the instrument's data (from 10 values) was reduced to a single value using PCA, it was used as a factor in a prediction model named PreSS (Predict Student Success $[8,41]$ ). The programming self-esteem value was the strongest factor in the prediction model throughout its development. The PreSS model uses three factors to predict student success: Bergin programming self-esteem, prior mathematical ability and number of hours per week a student plays computer games $[8,10,11]$. The PreSS model uses a machine learning algorithm to make the prediction. For a prediction model, reducing multiple values (measuring the same construct) to a single value (using the first component from the PCA) removes multicolinerarity which can improve prediction model performance in algorithms such as naïve Bayes (as it assumes that each attribute is independent) which was the algortithm used in the PreSS model [41, 43]. Detailed information on the factors, factor selection, data processing and the machine learning algorithm can be found in Bergin's work $[8,10,11]$.

In 2015-16, the BPSEI was used in a study investigating insights on gender differences in CS1 [44]. 693 students participated from 11 institutions, ten institutions in Ireland and one in Denmark. The goal of the study was to compare the profile of male $(n=547$, $78.9 \%)$ and female $(n=146,21.1 \%)$ students enrolled in CS1 courses, to determine if any significant differences could be identified by gender and between countries and institutions. The findings of this study included the BPSEI values, making this a valuable data set for comparison with upper secondary school teachers [44]. Most of these Irish institutions took part in an in-depth survey of CS1 offerings in another study which is a useful comparison for demographics and other specific details [5].

\subsection{Data Collection}

In our institution, we provide a suite of professional development workshops to upper secondary school teachers who teach Leaving Certificate Computer Science [45]. In this study the teacher cohort consisted of a mix of those who are currently teaching a new nationwide computer science upper second-level curriculum as well as teachers preparing to teach the curriculum in coming years. The teacher professional development (PD) survey was developed in 
2020 to record teacher's programming self-esteem before and after upper secondary school PD sessions. The instrument measures several factors including teachers' backgrounds, previous teaching experience and age. It also includes the BPSEI instrument as all of the PD sessions included programming in some form.

For the purpose of this study only the pre-PD programming selfesteem data was used (as post-session, perhaps our PD may have influenced their programming self-esteem, where future work aims to investigate this further). The CS1 students programming selfesteem was recorded at $\approx 4-6$ hours into their CS1 module as in [10]. With teachers teaching an upper secondary school formal computer science subject (or aspiring to), there is an assumption that the teachers had some level of prior programming experience (corroborated by the fact that only $5 \%$ of teachers reported that they did not have prior formal computing training). Thus recording teachers programming self-esteem at the earliest possible point in our interactions with them was pertinent, as research has shown that programming self-esteem is not temporally stable [44]. Therefore only teachers who were attending their first PD event with us were included in this study. From a total teacher cohort of 318 , after removing teachers who have been at a prior PD session, the total number of teachers in this study was reduced to 130 . Of these, we had a self-identified male:female ratio of $48: 52.36 \%$ of teachers were $40-59$ years of age, $46 \%$ were $20-39$, and $18 \%$ did not submit an age, as presented in Table 2. Geographically, the teachers who participated in this study teach the length and breadth of Ireland. This geographical spread is represented in Figure 1, where the circle radius shown correlates to the number of participants from that region.

Table 2: Teacher Age
Ranges.

\begin{tabular}{lrr}
\hline Age Range & N & \% \\
\hline Unreported & 23 & 18 \\
$18-29$ & 19 & 15 \\
$30-39$ & 40 & 31 \\
$40-49$ & 44 & 34 \\
$50-59$ & 3 & 0.02 \\
60 or over & 1 & 0.07 \\
\hline Total & $\mathbf{1 3 0}$ & $\mathbf{9 9}$
\end{tabular}

Figure 1: Geographical spread of teachers attending PD sessions.

\section{INVESTIGATING THE VALIDITY OF THE TEACHER PROGRAMMING SELF-ESTEEM SCALE}

We analysed the data from both scales using descriptive and statistical analysis. Results from the teacher PD survey were imported into Excel for analysis using Python. We investigated the programming self-esteem scale on the the teacher PD survey independently (compared with other survey variables) against the results in [44] using the BPSEI with CS1 students. Since the BPSEI was used in studies other than those predicting success in CS1, comparing the results of these two studies that use the same scale provides further evidence of criterion validity. Hence, we also compared the results of the BPSEI data collection from upper secondary school teachers PD with a study on insights on gender differences in CS1 that also uses the BPSEI instrument. We refer the reader to the work of Quille et al. [41, 44] for further information on demographics related to the CS1 students.

To analyse the data we needed to calculate the first principal component (using PCA) for the upper secondary school teachers, as this was the technique applied to the CS1 students. This resulted in a student/teacher with high programming self-esteem reporting a first principal component value that was in the negative region $(<0.00)$, while the students/teachers with low programming selfesteem reporting a first principal component value that was in the positive region $(>0.00)$. In both cases, approaching the upper and lower ranges suggested the highest or lowest programming self-esteem respectively.

For internal consistency of all items that are designed to measure a single construct such as the BPSEI, Cronbach's $\alpha$ can be used [18]. Cronbach's $\alpha$ values were compared to investigate if the BPSEI had an equivalent or greater internal consistency than the Rosenberg Self-Esteem questionnaire. The Cronbach's $\alpha$ values for the unmodified Rosenberg self-esteem questionnaire were in the range of 0.82 to 0.88 [47]. For the modified BPSEI during its development with undergraduate students on CS1, $\alpha$ was 0.91 , indicating good internal reliability $(n=102)[8,10,11]$. The programming self-esteem scale was evaluated for internal consistency using the 130 upper secondary school teacher responses, reporting a Cronbach's $\alpha=0.87$, indicating good internal reliability.

\section{COMPARING UPPER SECONDARY SCHOOL TEACHER AND CS1 SELF-ESTEEM}

Comparing domain-specific self-esteem using instruments that have participants self-report can provide additional insight into how the programming self-esteem scale for teachers should be changed in for future use. The nine month Teacher PD survey resulted in 318 participants and was administered between the 23rd March 2020 and the 16th December, 2020. Both the CS1 and upper secondary school teacher cohorts were surveyed using the BPSEI, however the CS1 students were given a four point Likert scale which was administered by the original author who developed the instrument [8].

The upper secondary school teachers were given a five point Likert scale with a neutral option was added (the remaining four points were identical). A five point scale was chosen because it allows respondents who genuinely have a neutral response to record this. Without this, such respondents may be forced to take either a positive or negative stance that may not be true and thus skew the results [27]. Also noted in [26] the risk of central tendency with the use of the neutral option on a Likert scale is reduced for those with higher cognitive skills (such as upper second level teachers). For these reasons the five point Likert scale was chosen for use on the upper secondary school teachers BPSEI.

In order to analyse these two scales using PCA analysis it was necessary to ensure both data sets were transformed to a numerical scale using linear transformation. We did this by normalizing 
the values between $0-1$, for both the teacher and CS1 student data. This is a valid interpretation of these different scales according the Bishop and Herron in "Use and Misuse of the Likert Item Responses and Other Ordinal Measures" [12]. Here Bishop explains that any linear transformation can be made without impacting the data or its analysis. The PCA technique which calculated the first principal component, was conducted on this normalised data. The PCA (programming self-esteem) values were then calculated for the combined normalized data set $(n=823)$, and the findings are presented in Table 3.

Table 3: Upper Secondary School teachers vs. CS1 Undergraduates Self-Esteem. Negative values reflect a higher selfesteem.

\begin{tabular}{lrrr}
\hline Group & Self-Esteem & SD & N \\
\hline Secondary Teachers & +0.3367 & 0.2028 & 130 \\
CS1 students & -0.0631 & 0.3964 & 693 \\
\hline
\end{tabular}

Using the same statistical techniques as the CS1 study [44], a Welch's $t$-test (with $95 \%$ confidence level) was used to test for statistical significance [50]. This is more suitable than a Student's $t$-test as the data sets (or subsets such as reported gender data) have unequal variance. The difference between teachers' and CS1 students' self esteem was statistically significant $(p<0.0001)$ with teachers reporting a lower programming self-esteem compared to CS1 undergraduate students. Perhaps this is due to CS1 students self-selecting the programme, where typically teachers are trained in other disciplines such as mathematics or science, and this alone warrants further investigation. Putting aside the underlying cause for a moment, this is still a finding of high value, as approaches must be included (if not already) to try to improve teacher programming self-esteem during PD. Previous studies have already shown that teachers sometimes avoid PD labelled as a more difficult topic [45], and perhaps this is due to teacher programming self-esteem being lower than that of undergraduate CS1 students. Attention should be given to this finding in two directions: first when developing PD language/content and second, how this is presented to upper secondary school teachers prior to the PD.

Next, an investigation into differences in programming selfesteem was conducted by gender. Previous research in CS1 has shown that female students exhibit a lower programming selfesteem than their male counterparts [44]. The results comparing upper second level teachers and CS1 students by gender are presented in Table 4. A statistically significant difference was reported between group means as determined by one-way $\operatorname{ANOVA}(\mathrm{F}(3$, $819)=60.13 \mathrm{p}<0.0001$ ), reporting that not only is there a statistically significant difference between teachers and CS1 students, but differences may also exist by gender.

A Tukey HSD post-hoc test was also performed to conduct a deeper analysis of the differences between gender and cohorts, with the findings are presented in Table 5 .

While the difference in the mean reported in Table 4 is statistically significant and as expected for female and male CS1 students, the same cannot be said for teachers. Female teachers and male
Table 4: Upper Secondary School teachers vs. CS1 Undergraduates Self-Esteem by Gender. Negative values reflect a higher self-esteem.

\begin{tabular}{lrrr}
\hline Group & Self-Esteem & SD & N \\
\hline Teachers Female & +0.31095 & 0.16849 & 68 \\
Teachers Male & +0.36494 & 0.23151 & 62 \\
CS1 Female & +0.11865 & 0.40053 & 146 \\
CS1 Male & -0.11169 & 0.38091 & 547 \\
\hline
\end{tabular}

Table 5: Upper Secondary School Teachers vs. CS1 Undergraduates by Gender, Tukey HSD post-hoc analysis with $p$ values (CI 95\%).

\begin{tabular}{lrrrr}
\hline & Teachers F & Teachers M & CS1 F & CS1 M \\
\hline Teachers Female & - & 0.8314 & 0.0018 & $<0.0001$ \\
Teachers Male & 0.8314 & - & $<0.0001$ & $<0.0001$ \\
CS1 Female & 0.0018 & $<0.0001$ & - & $<0.0001$ \\
CS1 Male & $<0.0001$ & $<0.0001$ & $<0.00001$ & - \\
\hline
\end{tabular}

teachers have reported no statistically significant difference in programming self-esteem. This differs from other research [54], which reported that male teachers programming self-esteem was greater than that of female teachers across K-12.

\section{DISCUSSION}

This study provides evidence of criterion validity for using the teacher programming self-esteem scale, by comparing it to the same scale used for CS1 students. In addition, evidence of reliability in the form of internal consistency was found using Cronbach's alpha $(\alpha)$, for the use of this scale with upper secondary school computer science teachers (reporting $\alpha=0.87$ ). This study reported a similar $\alpha$ value to that reported when the scale was used with CS1 students (CS1 students $\alpha=0.91)$. We consider these results to provide indicative evidence of validity and reliability for the use of the BPSEI scale with upper secondary school teachers.

With respect to the related construct, programming self-efficacy, it has previously been shown through a meta-analysis in computing that females score lower on these scales due to their underestimation of their computing abilities [49]. Self-esteem has also been shown that women majoring in computing have lower self-esteem in the field than in general and that their view of their worth of knowledge in computing was low, despite understanding the concept(s) $[24,25]$. Females in the CS1 student cohort in this study reported significantly lower self-esteem than their male peers [44]; however, while differences in gender exist with CS1 students, no such differences were found between female and male upper secondary school teachers. This was not the case with K-12 teachers in a recent international study [54]. Future work will be to investigate why such differences exist between CS1 male and female students and and not male and female upper secondary school teachers. The findings from this study support those found in [31] - that results of teacher self-efficacy influences the type of professional development considerations that must be made. A "one-size-fits-all" approach should not be taken and care should be given to content 
delivery and scaffolding. Mannila, et al. also call for different levels of support and guidance for teachers with low, medium, and high self-efficacy based on digital competencies evaluated [31].

A recent study in Australia found that there were differences in teacher self-efficacy according to technologies and standards related to phases of curriculum implementation, with teachers further along in this process having higher self-efficacy [53]. However in a wider context this is concerning, as teachers at upper secondary school teaching or considering to teach the second level capstone computer science subject report statistically significantly lower programming self-esteem to that CS1.

Given this current and previous research, creating PD to match various levels of programming self-esteem and self-efficacy in content knowledge and pedagogical content knowledge may be important for teachers to have a successful PD experience and to raise their self-esteem, thereby influencing higher academic achievement among their students [24, 25, 35, 52]. With previous research indicating that self-esteem can be raised through training, this could be embedded into PD curriculum so that teachers leave with higher self-esteem. Perhaps most importantly, we would hope that teachers with higher self-esteem would result in better learning as well as students with higher self-esteem.

\subsection{Limitations}

Although this study had a smaller number of participants (130) than the study we compared our results to (several hundred), the statistical power of our comparisons should not be an issue as both participant numbers are on the same order of magnitude. Further, both the CS1 study and this study were conducted within the same educational system, broadly speaking. While this may add validity for the comparison in this study under an jurisdictional educational context, it may not translate to other systems or jurisdictions.

For this study only pre-PD survey responses were used from teachers attending their first PD event, where successive teacher occurrences were removed. Although we do not have exact information on the prior programming experience for these teachers, we know that $95 \%$ of the teachers in this study had prior computer science experience, making it reasonable to think that they might have higher programming self-esteem than CS1 students. However, it could be that some teachers' last prior CS experience was several years ago. Future work will include collecting additional details on their prior experience. In addition, as some of the teachers were already teaching programming (and this particular second-level curriculum is quite close to CS1) we would again find it reasonable if they had higher self-esteem to begin with. Nonetheless, this is not what we found and warrants further investigation.

Though CS1 may be required of some students, it is also somewhat self-selecting. Students selecting computing as a major would naturally take CS1 and have a good chance of being interested in the area. Students with prior experience could be more interested in the subject due to previous success within those experiences, which has been shown to be positively correlated to self-efficacy [14]. Teachers, however, may not be choosing to teach computing and may be required to learn and teach computing by their administration. This could have influenced our study. Unlike self-efficacy, self-esteem in general is more fluid in childhood and becomes more fixed in adulthood [1]. The self-selection, then, may be indicating that the students' learning is more fixed due to their prior experiences and positive outlook, while more fluid in the teachers as they come to terms with learning the content. Additionally, studying a subject as a student, and teaching it as part of a job, are not symmetric experiences. There are many pressures on teachers that students do not face, many of them more public and which impact more people by their very definition. This may lead to stress, and partially explain lower self-esteem in teachers compared to students.

\section{CONCLUSION AND FUTURE WORK}

As $\mathrm{K}-12$ computing education becomes more established and the demand to train teachers in content knowledge and pedagogical content knowledge increases, the value of properly understanding the role programming self-esteem plays in their ability to learn and teach is increasingly important. In this study we demonstrated the validity of the BPSEI scale for CS1 students as a suitable tool for measuring the same in teachers. We found that teachers have lower self-esteem than CS1 students, in a similar educational system. We also found that teachers identifying as female have similar programming self-esteem to their counterparts identifying as male. This differs to prior studies $[44,54]$ and warrants future investigation.

This study yielded future research questions:

- Is there a difference between teacher and student programming self-esteem in other contexts, and if so, why?

- What is the reason for the similarities in the male and female teachers programming self-esteem given other studies in the area have found significant differences between these groups?

- Is there a correlation between students' academic achievement and their teacher's programming self-esteem?

- How can we improve teacher programming self-esteem?

- Are there other fields in which a gap in teacher self-esteem differs from those of undergraduate students in the introductory course of that field?

- Can we create a similar prediction model to PreSS (Predict Student Success) to help predict teacher success in the classroom with the goal of informing professional development efforts?

We will continue to build on this research, reflect further on how teacher self-esteem in programming affects academic achievement among students, and how professional development curricula can be improved to help increase teacher programming self-esteem.

\section{REFERENCES}

[1] Joseph A Bailey 2nd. 2003. The foundation of self-esteem. fournal of the National Medical Association 95, 5 (2003), 388.

[2] Albert Bandura. 1982. Self-efficacy mechanism in human agency. American psychologist 37, 2 (1982), 122.

[3] Albert Bandura. 2006. Guide for constructing self-efficacy scales. Self-efficacy beliefs of adolescents 5, 1 (2006), 307-337.

[4] Nathan Bean, Joshua Weese, Russell Feldhausen, and R Scott Bell. 2015. Starting from scratch: Developing a pre-service teacher training program in computational thinking. In Frontiers in Education Conference (FIE). IEEE, 1-8.

[5] Brett A. Becker. 2019. A Survey of Introductory Programming Courses in Ireland. In Proceedings of the 2019 ACM Conference on Innovation and Technology in Computer Science Education (Aberdeen, Scotland Uk) (ITiCSE '19). ACM, New York, NY, USA, 58-64. https://doi.org/10.1145/3304221.3319752

[6] Brett A Becker and Catherine Mooney. 2016. Categorizing compiler error messages with principal component analysis. In 12th China-Europe International 
Symposium on Software Engineering Education (CEISEE 2016), Shenyang, China, 28-29 May 2016

[7] Brett A. Becker and Keith Quille. 2019. 50 Years of CS1 at SIGCSE: A Review of the Evolution of Introductory Programming Education Research. In Proceedings of the 50th ACM Technical Symposium on Computer Science Education (Minneapolis, MN, USA) (SIGCSE '19). Association for Computing Machinery, New York, NY, USA, 338-344. https://doi.org/10.1145/3287324.3287432

[8] Susan Bergin. 2006. A computational model to predict programming performance. Ph.D. Dissertation. Department of Computer Science, Maynooth University.

[9] Susan Bergin, Aidan Mooney, John Ghent, and Keith Quille. 2015. Using machine learning techniques to predict introductory programming performance. International fournal of Computer Science and Software Engineering (IFCSSE) 4, 12 (2015), 323-328.

[10] Susan Bergin and Ronan Reilly. 2005. Programming: Factors That Influence Success. SIGCSE Bull. 37, 1 (February 2005), 411-415. https://doi.org/10.1145/ 1047124.1047480

[11] Susan Bergin and Ronan Reilly. 2006. Predicting introductory programming performance: A multi-institutional multivariate study. Computer Science Education 16, 4 (2006), 303-323.

[12] Phillip A Bishop and Robert L Herron. 2015. Use and misuse of the Likert item responses and other ordinal measures. International journal of exercise science 8 , 3 (2015), 297.

[13] Janet Seeley Blouin. 2011. High school seniors' computer self-efficacy and interest in computer science careers. Ph.D. Dissertation. University of Georgia.

[14] Tor Busch. 1996. Gender, group composition, cooperation, and self-efficacy in computer studies. Fournal of educational computing research 15, 2 (1996), 125-135.

[15] Stephen Colgan, Keith Quille, Jelena Vasic, and Seán McHugh. 2019. Predict ing Student Success. Early for a VTOS Student.. In International Conference on Engaging Pedagogy (ICEP) (ICEP').

[16] Daan Creemers, Ron Scholte, Rutger Engels, Mitchell Prinstein, and Reinout W Wiers. 2013. Damaged self-esteem is associated with internalizing problems. Frontiers in psychology 4 (2013), 152.

[17] Lee J Cronbach and Paul E Meehl. 1955. Construct validity in psychological tests. Psychological bulletin 52, 4 (1955), 281-302.

[18] L. J. Cronbach and Paul E Meehl. 1962. Psychological bulletin. Psychological bulletin 59, 4 (1962), 257-72. http://www.ncbi.nlm.nih.gov/pubmed/19586159

[19] Natalie Culligan, Keith Quille, and Susan Bergin. 2016. VEAP: A Visualisation Engine and Analyzer for preSS\#. In Proceedings of the 16th Koli Calling International Conference on Computing Education Research (Koli, Finland) (Koli Calling '16) ACM, New York, NY, USA, 130-134. https://doi.org/10.1145/2999541.2999553

[20] Adrienne Decker and Monica M McGill. 2019. A Topical Review of Evaluation Instruments for Computing Education. In Proceedings of the 50th ACM Technical Symposium on Computer Science Education. ACM, 558-564.

[21] Rosemary Dobbins. 1996. Student teacher self-esteem in the practicum. Australian fournal of Teacher Education 21, 2 (1996), 2.

[22] Ann W Drobnis. 2010. Girls in computer science: A female only introduction class in high school. Ph.D. Dissertation.

[23] Philip Guo. 2014. Python is Now the Most Popular Introductory Teaching Language at Top US Universities. Communications of the ACM Blog (BLOG@CACM), fuly (2014). https://cacm.acm.org/blogs/blog-cacm/176450-python-is-now-themost-popular-introductory-teaching-language-at-top-u-s-universities

[24] Rachelle Kristof Hippler. 2018. Understanding the Exodus of Undergraduate Women from Computer Science Majors: A Two-Factor Model of Self-Esteem. In Proceedings of the Technology, Mind, and Society. ACM, 16.

[25] Rachelle Kristof Hippler. 2019. Overcoming Doubt: Workshop Aimed at Giving Students Strategies to Build Their Authentic Self-Esteem Within Their Computing Major. In Proceedings of the 50th ACM Technical Symposium on Computer Science Education (Minneapolis, MN, USA) (SIGCSE '19). ACM, New York, NY, USA 1258-1258. https://doi.org/10.1145/3287324.3293839

[26] Jon A Krosnick, Allyson L Holbrook, Matthew K Berent, Richard T Carson, W Michael Hanemann, Raymond J Kopp, Robert Cameron Mitchell, Stanley Presser Paul A Ruud, V Kerry Smith, et al. 2002. The impact of "no opinion" response options on data quality: non-attitude reduction or an invitation to satisfice? Public Opinion Quarterly 66, 3 (2002), 371-403.

[27] Jon A. Krosnick and Stanley Presser. 2010. Handbook of Survey Research, Second Edition. Vol. 2. Emerald Group Publishing Limited.

[28] Jihyun Lee and Valerie J Shute. 2010. Personal and social-contextual factors in K-12 academic performance: An integrative perspective on student learning. Educational Psychologist 45, 3 (2010), 185-202.

[29] Elizabeth A Linnenbrink and Paul R Pintrich. 2002. Motivation as an enabler for academic success. School psychology review 31, 3 (2002), 313-327.

[30] Riia Luhtanen and Jennifer Crocker. 1992. A collective self-esteem scale: Selfevaluation of one's social identity. Personality and social psychology bulletin 18, 3 (1992), 302-318.

[31] Linda Mannila, Lars-Ake Nord'en, and Arnold Pears. 2018. Digital Competence, Teacher Self-Efficacy and Training Needs. In ACM International Computing Education Research Workshop. https://doi.org/10.1145/3230977.3230993
[32. Monica M. McGill and Jeffrey Xavier. 2019. Measuring Reliability and Validity. Self-efficacy on the Students' Motivation and Achievement. Theory \& Practice in Language Studies 2, 3 (2012).

[34] Catherine Mooney and Brett A. Becker. 2021. Investigating the Impact of the COVID-19 Pandemic on Computing Students' Sense of Belonging. In Proceedings of the 52nd ACM Technical Symposium on Computer Science Education (Virtual Event, USA) (SIGCSE '21). Association for Computing Machinery, New York, NY, USA, 612-618. https://doi.org/10.1145/3408877.3432407

[35] Ann Quade. 2003. Development and validation of a computer science self-efficacy scale for CS0 courses and the group analysis of CS0 student self-efficacy. In Proceedings ITCC 2003. International Conference on Information Technology: Coding and Computing. IEEE, 60-64.

[36] Keith Quille. 2019. Predicting and Improving Performance on Introductory Programming Courses (CS1). Ph.D. Dissertation. National University of Ireland Maynooth. http://mural.maynoothuniversity.ie/12840/

[37] K Quille and S Bergin. 2015. Programming: Factors that Influence Success Revisited and Expanded. In International Conference on Enguaging Pedagogy (ICEP), 3rd and 4th December, College of Computing Technology, Dublin, Ireland.

[38] Keith Quille and Susan Bergin. 2016. Does Scratch improve self-efficacy and performance when learning to program in C\#? An empirical study.. In International Conference on Enguaging Pedagogy (ICEP).

[39] Keith Quille and Susan Bergin. 2016. Programming: Further Factors that Influence Success. In PPIG. 14.

[40] Keith Quille and Susan Bergin. 2018. Programming : Predicting Student Success Early in CS1. A Re-validation and Replication Study. In 23rd Annual ACM Conference on Innovation and Technology in Computer Science Education (ITiCSE'18). $15-20$.

[41] Keith Quille and Susan Bergin. 2019. CS1: how will they do? How can we help? A decade of research and practice research and practice. Computer Science Education 29 (2019), 254-282. https://doi.org/10.1080/08993408.2019.1612679

[42] Keith Quille and Susan Bergin. 2020. Promoting a Growth Mindset in CS1: Does One Size Fit All? A Pilot Study. In Proceedings of the 2020 ACM Conference on Innovation and Technology in Computer Science Education (Trondheim, Norway) (ITiCSE '20). Association for Computing Machinery, New York, NY, USA, 12-18. https://doi.org/10.1145/3341525.3387361

[43] Keith Quille, Susan Bergin, and Aidan Mooney. 2015. PreSS \#, A Web-Based Educational System to Predict Programming Performance. International fournal of Computer Science and Software Engineering (IFCSSE) 4 (2015), 178-189.

[44] Keith Quille, Natalie Culligan, and Susan Bergin. 2017. Insights on Gender Differences in CS1: A Multi-institutional, Multi-variate Study.. In Proceedings of the 2017 ACM Conference on Innovation and Technology in Computer Science Education (Bologna, Italy) (ITiCSE '17). ACM, New York, NY, USA, 263-268. https://doi.org/10.1145/3059009.3059048

[45] Keith Quille, Roisin Faherty, Susan Bergin, and Brett A. Becker. 2018. Second Level Computer Science: The Irish K-12 Journey Begins. In Proceedings of the 18th Koli Calling International Conference on Computing Education Research (Koli, Finland) (Koli Calling '18). ACM, New York, NY, USA, Article 22, 5 pages. https: //doi.org/10.1145/3279720.3279742

[46] Vennila Ramalingam and Susan Wiedenbeck. 1998. Development and validation of scores on a computer programming self-efficacy scale and group analyses of novice programmer self-efficacy. Journal of Educational Computing Research 19, 4 (1998), 367-381.

[47] Morris Rosenberg. 1965. Society and the adolescent self-image. Vol. 11. Princeton university press Princeton, $\mathrm{NJ}$

[48] Harun Sahin. 2017. Emotional Intelligence and Self-Esteem as Predictors of Teacher Self-Efficacy. Educational Research and Reviews 12, 22 (2017), 1107-1111.

[49] Jo Sanders. 2005. Gender and technology in education: A research review. Seattle: Center for Gender Equity. Bibliography retrieved March 20 (2005), 2006.

[50] Shlomo S Sawilowsky. 2002. Fermat, Schubert, Einstein, and Behrens-Fisher: The Probable Difference Between Two Means When $\sigma 1^{\wedge} 2 \neq \sigma \_2^{\wedge} 2$. Fournal of Modern Applied Statistical Methods 1, 2 (2002), 55.

[51] Hemant Lata Sharma and Gunjan Nasa. 2014. Academic self-efficacy: a reliable predictor of educational performances. British fournal of Education 2, 3 (2014), $57-64$.

[52] Gholamreza Torkzadeh and Xenophon Koufteros. 1994. Factorial validity of a computer self-efficacy scale and the impact of computer training. Educational and psychological measurement 54, 3 (1994), 813-821.

[53] Rebecca Vivian and Katrina Falkner. 2018. A survey of Australian teachers' self-efficacy and assessment approaches for the K-12 digital technologies curriculum. In Proceedings of the 13th Workshop in Primary and Secondary Computing Education. ACM, 5.

[54] Rebecca Vivian, Keith Quille, Monica M. McGill, Katrina Falkner, Sue Sentance, Sarah Barksdale, Leonard Busuttil, Elizabeth Cole, Christine Liebe, and Francesco Maiorana. 2020. An International Pilot Study of K-12 Teachers' Computer Science Self-Esteem (ITiCSE '20). Association for Computing Machinery, New York, NY, USA, 117-123. https://doi.org/10.1145/3341525.3387418 